\title{
Breaking properties of neutron star crust
}

\author{
D.A. Baiko* and A.I. Chugunov† \\ Ioffe Institute, Politekhnicheskaya 26, 194021 Saint Petersburg, Russian Federation
}

Accepted; Received ; in original form

\begin{abstract}
The strength of neutron star crust is crucial for modelling magnetar flares, pulsar glitches and gravitational wave emission. We aim to shed some light on this problem by analysing uniaxial stretch deformation (elongation and contraction) of perfect bodycentered cubic Coulomb crystals, paying special attention to the inherent anisotropy of this process. Our analysis is based on the semi-analytical approach of Baiko \& Kozhberov (2017), which, for any uniform deformation, allows one to calculate, in fully non-linear regime, critical deformation parameters beyond which the lattice loses its dynamic stability. We determine critical strain, pressure anisotropy and deformation energy for any stretch direction with respect to the crystallographic axes. These quantities are shown to be strongly anisotropic: they vary by a factor of almost 10 depending on the orientation of the deformation axis. For polycrystalline crust, we argue that the maximum strain for the stretch deformation sustainable elastically is 0.04 . It is lower than the breaking strain of 0.1 obtained in molecular dynamic simulations of a shear deformation by Horowitz \& Kadau (2009). The maximum pressure anisotropy of polycrystalline matter is estimated to be in the range from 0.005 to $0.014 n Z^{2} e^{2} / a$, where $n$ is the ion number density, $Z e$ is the ion charge, and $a$ is the ion-sphere radius. We discuss possible mechanisms of plastic motion and formation of large crystallites in neutron star crust as well as analyse energy release associated with breaking of such crystallites in the context of magnetic field evolution and magnetar flaring activity.
\end{abstract}

Key words: dense matter - stars: neutron.

\section{INTRODUCTION}

The crust of a neutron star (NS) is crystallised, i.e. ions (atomic nuclei) form a lattice (e.g., Haensel, Potekhin \& Yakovlev 2007; Chamel \& Haensel 2008; Caplan \& Horowitz 2017). In most of the crust the groundstate lattice has the body-centered cubic (bcc) structure (see Baiko 2014 for discussion of screening effects). Crystalline properties of NS crust are invoked to explain a number of observed astrophysical phenomena. For instance, crustal elasticity is thought to be important for interpretation of quasi-periodic oscillations observed in magnetars (e.g., Gabler, Cerdá-Durán, Stergioulas, Font \& Müller 2018). Solid crust can support asymmetric distributions of density, so-called, mountains, which can produce gravitational wave $(\mathrm{GW})$ emission (Ushomirsky, Cutler \& Bildsten 2000; Horowitz 2010; Johnson-McDaniel \& Owen 2013). Size of these mountains is limited by the strength of the crust (i.e. maximum stress, which the elastically deformed crustal material can withstand). If the crust is indeed as strong as it is suggested by molecular dynamic (MD) sim-

* E-mail:baiko@astro.ioffe.ru

† E-mail:andr@astro.ioffe.ru ulations (Horowitz \& Kadau 2009; Chugunov \& Horowitz 2010, 2012), the GW emission can be poweful enough to be detectable by existing GW observatories (Johnson-McDaniel 2013; Caplan \& Horowitz 2017). Haskell \& Patruno (2017) have recently suggested that this GW emission could be responsible for observed spin-down during the accreting phase of PSR J1023+0038. Crust breaking under magnetic stress is likely responsible for magnetar outbursts (e.g., Li, Levin \& Beloborodov 2016), while plastic motion may be crucial for magnetic field evolution (e.g., Lander 2016). Tsang et al. (2012) argued that crust breaking could produce an electromagnetic precursor of the GW signal from NS mergers.

Thus, an accurate knowledge of the crust strength is fundamental for NS physics and in this paper we analyse it, paying special attention to its anisotropy. Previous works in this field explored a shear deformation by MD simulation (Horowitz \& Kadau 2009; Chugunov \& Horowitz 2010, 2012; Hoffman \& Heyl 2012) and volume-conserving crystal stretching in two highly symmetric directions semianalytically (Baiko \& Kozhberov 2017; Paper 1). Here we apply the approach proposed in the latter work to study stretching in arbitrary directions.

In Paper 1, the existence of a limit of a uniform 
Coulomb crystal deformation, above which the crystal loses dynamic stability, has been demonstrated using standard lattice dynamics. The maximum crystal elongation and contraction factors as well as the maximum pressure anisotropy at breaking has been predicted essentially analytically. The authors have discussed two particular stretch directions and have found that the limiting stretch factor and the pressure anisotropy were strongly dependent on the angle between the stretch direction and the crystallographic axes.

In this paper, we generalise the results of Paper 1 and perform an extensive study of the dependence of the maximum deformation, pressure anisotropy and deformation energy on the stretch direction for a perfect bcc Coulomb crystal (Section 2). We find this dependence to be very strong (for instance, critical pressure anisotropy differs by a factor of almost 10 between the weakest and the strongest directions). We point out that the maximum deformation is typically smaller for a contraction of the lattice, than for an elongation. Also, typical maximum contraction deformation is about $5 \%$, which is a factor of two lower than the breaking strain for the shear deformation, obtained by Horowitz \& Kadau (2009) and Chugunov \& Horowitz (2010, 2012). Our results clearly demonstrate that the Tresca and von Misses failure criteria are not valid for perfect Coulomb lattice in NS crust. Our limits of the lattice stability are upper bounds for particular deformation types, because other factors, such as ion motion about lattice nodes (due to zero-point or finite temperature effects) or electron screening, will further degrade material strength.

In Subsection 2.1 we calculate the elastic energy at the critical deformation for all stretch directions.

In Section 3 we consider astrophysical applications of our results. In particular, Subsection 3.2 is devoted to breaking properties of polycrystalline matter, which is a possible microscopic state of NS crust. In Subsection 3.4 we estimate heat sources associated with breaking events in magnetar crust.

Our results can be applied to both outer and inner crust of neutron stars provided that the effect of dripped neutrons on the interionic interaction can be neglected, and excluding the exotic pasta phases, which may appear at mass densities exceeding $10^{14} \mathrm{~g} \mathrm{~cm}^{-3}$.

\section{CRUSTAL MODEL, SCALINGS AND BREAKING PROPERTIES}

We model NS crust as a deformed bcc Coulomb crystal composed of identical ions. This means that ions interact with each other via pure Coulomb forces and we neglect screening of this interaction by electrons. It is a widely employed approximation in NS literature (e.g., Haensel et al. 2007; Chamel \& Haensel 2008). In particular, it is known to predict elastic properties of the crust with reasonable accuracy (Baiko 2011, 2015). Chugunov \& Horowitz (2012) have analysed effects of electron screening on the breaking stress for the shear deformation and have demonstrated that screening reduced strength, but not very much. Thus, we expect that an accurate treatment of electron screening can only reduce maximum pressure anisotropy. We discuss stability of a deformed static lattice and do not include any effects associated with ion motion, which are expected to further weaken critical deformation properties of the lattice (e.g., Regel', Slutsker \& Tomashevskii 1972).

We are interested in the maximum deformations for arbitrary stretch directions. However, the symmetry of the lattice allows one to restrict themselves by deformations in a narrower solid angle which contains all essentially different directions of the crystal stretch. Maximum deformations for all other directions can then be obtained by simple symmetry transformations. For the bcc lattice, it means studying stretch directions characterised by unit vectors $\boldsymbol{s}=\left(s_{x}, s_{y}, s_{z}\right)$ satisfying $s_{x} \geqslant s_{y} \geqslant s_{z} \geqslant 0$, where $x y z$ is a Cartesian reference frame aligned with the bcc lattice cube. We specify these directions by two angles, $\theta$ and $\phi$, where $\theta$ is the angle between the stretch direction and the $x$-axis, while $\phi$ is the azimuthal angle in the plane perpendicular to the $x$-axis. Angle $\phi$ varies from 0 [e.g., for direction $(1,1,0) / \sqrt{2}$ ] to $\pi / 4$ [e.g., for direction $(1,1,1) / \sqrt{3}$ ]. Angle $\theta$ varies from 0 to $\tan ^{-1}(1 / \cos \phi)$.

The stretches under study are volume-conserving deformations of the perfect lattice, in which all projections of the lattice vectors along the stretch axis are multiplied by a factor $1+\epsilon$, while all projections in the plane perpendicular to this direction are multipled ${ }^{1}$ by $1 / \sqrt{1+\epsilon}$. Alternatively (as in Paper 1), all projections of the lattice vectors along the stretch axis can be multiplied by a factor $\xi$ and then an overall scale factor $\xi^{-1 / 3}$ is apllied to preserve the volume. Thus $\xi=(1+\epsilon)^{3 / 2}$. Clearly, an elongation corresponds to $\epsilon>0$ or $\xi>1$, while a contraction is obtained in the opposite case. The maximum deformation is determined for each direction $(\theta, \phi)$ independently for both elongation and contraction. It corresponds to a minimum value of $|\epsilon|$, at which the lattice is no longer stable or, to be more precise, at which a zero frequency phonon mode appears. To locate this transition, we calculate the dynamic matrix of the deformed Coulomb lattice using the Ewald transformation and solve the respective secular equation on a dense grid of wavevectors $\boldsymbol{k}$ in the first Brillouin zone. For all considered deformations, the unstable mode appears at $k \rightarrow 0$, indicating that the loss of stability takes place on large spatial scales.

For certain non-volume-conserving deformations we can adopt a two-step procedure in which the first step is a uniform compression/expansion to the desired density followed by a volume-conserving stretch to the final state. Since the first deformation preserves bcc lattice, it is always stable. Thus the total deformation will be stable if and only if the second deformation does not lead to the loss of stability.

In the Coulomb crystal, the electrostatic part of the stress tensor $\tilde{\sigma}_{i j}$ has a simple scaling with density and composition:

$$
\tilde{\sigma}_{i j}=\frac{n Z^{2} e^{2}}{a} \sigma_{i j},
$$

where the dimensionless stress tensor $\sigma_{i j}$ depends only on deformation, i.e. on $\theta, \phi$, and $\xi$. In this case, $n$ is the ion number density, $Z$ is the ion charge number, $e$ is the elementary charge, and $a=(4 \pi n / 3)^{-1 / 3}$ is the ion-sphere radius. For non-deformed bcc lattice, $\sigma_{i j}=-\zeta \delta_{i j} / 3$, where $\zeta \approx$

1 For small deformations, the Cauchy's strain tensor $u_{i j}$ is diagonal in the reference frame, where axis 3 and the stretch axis coincide, $u_{11}=u_{22}=-\epsilon / 2 ; u_{33}=\epsilon$. 


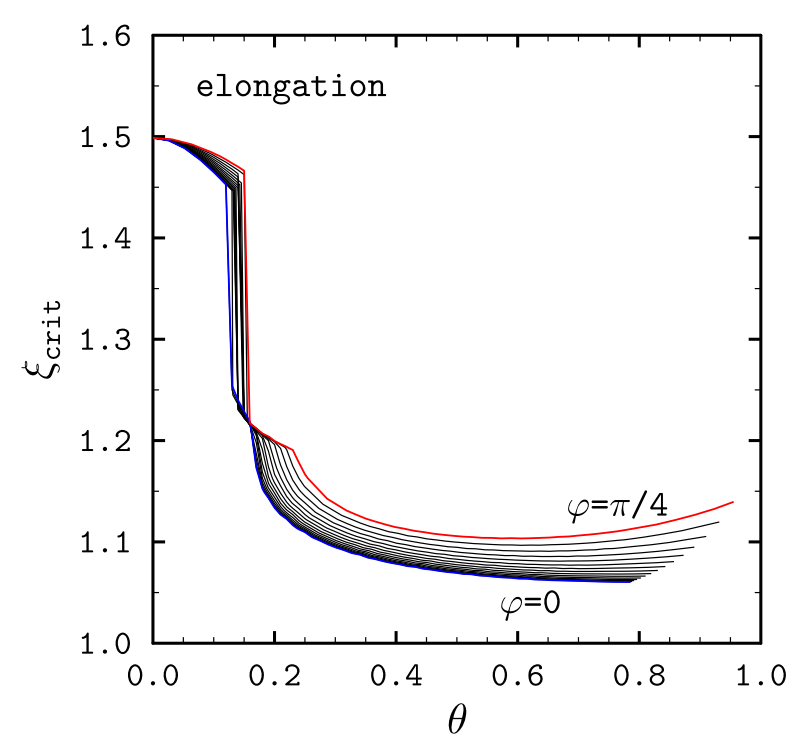

Figure 1. Critical stretch factor for elongation.

-0.8959293 is the Madelung constant (e.g., Haensel et al. 2007).

For deformed lattice, the stress tensor remains symmetric $\left(\sigma_{i j}=\sigma_{j i}\right.$, see, e.g., Landau, Lifshitz, Kosevich, Sykes, Pitaevskiı̌ \& Reid 1986) and thus can be always cast in the diagonal form. However, it becomes anisotropic (i.e. $\sigma_{i j} \neq \sigma \delta_{j i}$ ) and we, in analogy with the Tresca criterion, characterise it by the difference between the maximum and the minimum eigenvalues of the stress tensor, which we refer to as pressure anisotropy $\Delta \tilde{P}$. According to (1),

$$
\Delta \tilde{P}=\frac{n Z^{2} e^{2}}{a} \Delta P
$$

where $\Delta P$ is the dimensionless pressure anisotropy.

The excess energy of the deformed lattice with respect to the non-deformed configuration can be written as

$$
\Delta \tilde{U}=\frac{N Z^{2} e^{2}}{a} \Delta U
$$

where $N$ is the number of ions. Dimensionless quantities $\Delta P$ and $\Delta U$ depend neither on density nor on composition being functions of deformation only. Note also, that $\Delta U$ is the excess energy per ion in units of $Z^{2} e^{2} / a$.

Below we present the values of $\Delta P_{\text {crit }}$ and $\Delta U_{\text {crit }}$ to characterise lattice properties at the onset of lattice instability. Scalings (2) and (3) allow one to recalculate the respective properties in physical units for arbitrary density and composition.

Our results for critical stretch factors $\xi_{\text {crit }}$ are summarised in Fig. 1 for elongation and Fig. 2 for contraction. In both cases we show the dependence of the critical stretch factor on $\theta$ for 16 values of $\phi$ equally spaced between 0 (blue curve) and $\pi / 4$ (red curve). In Figs. 3 and 4 we show critical pressure anisotropy $\Delta P_{\text {crit }}$ for elongation and contraction, respectively, as a function of the same angles. This quantitity is calculated using the Ewald transformation for the critically deformed Coulomb lattice.

Let us start with a discussion of elongation. One sees that the critical strain strongly depends on $\theta$ : for $\theta \lesssim 0.2$, it can be as large as $\epsilon_{\text {crit }} \approx 0.3\left(\xi_{\text {crit }} \approx 1.5\right)$, but for $\theta \gtrsim 0.4$,

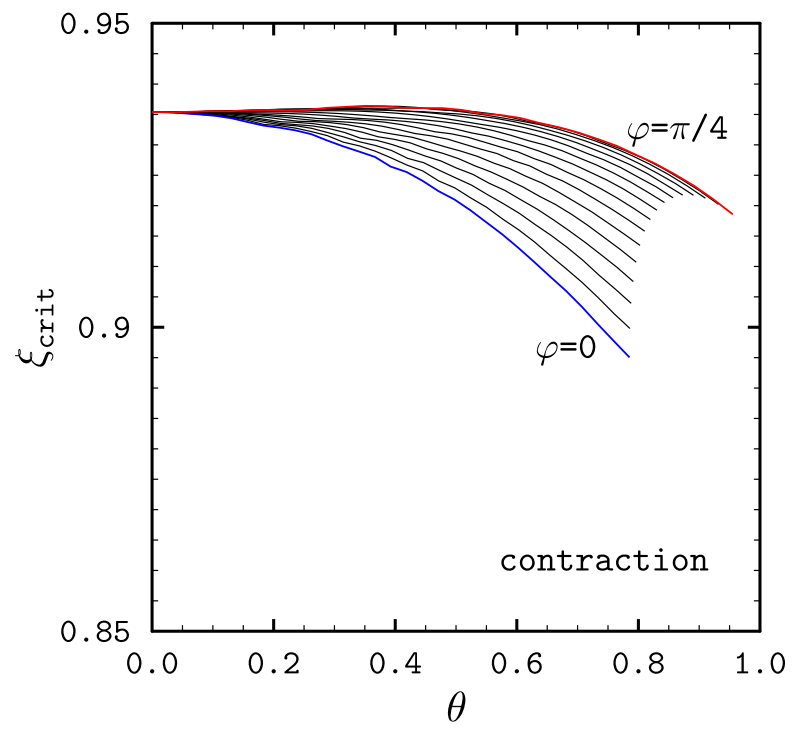

Figure 2. Critical stretch factor for contraction.

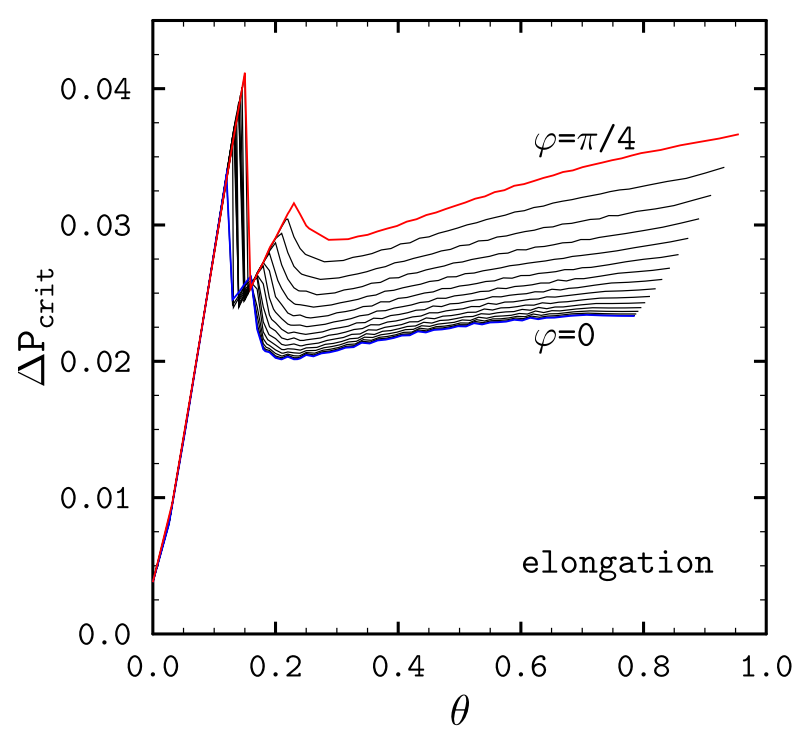

Figure 3. Dimensionless critical pressure anisotropy for elongation.

$\epsilon_{\text {crit }} \lesssim 0.07$ ( $\left.\xi_{\text {crit }} \lesssim 1.1\right)$. This is 1.4 times smaller than the critical strain $\epsilon_{\text {crit }}^{\mathrm{MD}} \approx 0.1$ obtained in MD simulations of the shear deformation (Horowitz \& Kadau 2009). Weakness (i.e. loss of stability at small $\Delta P$ ) of the Coulomb lattice for stretching along the cube edge $(\theta=0)$ was demonstrated in Paper 1; our results reveal that it takes place in a rather narrow solid angle. The critical pressure anisotropy $\Delta P_{\text {crit }}$ rapidly increases with increase of $\theta$. For $\theta \gtrsim 0.1, \Delta P_{\text {crit }}$ varies within a factor of $2\left(\Delta P_{\text {crit }}=0.02-0.04\right)$. In the vicinity of the global maximum, it reaches the breaking stress for the shear deformation of a perfect crystal, $\Delta P^{\mathrm{MD}} \approx 0.039$, obtained by Chugunov \& Horowitz (2010) at $T \rightarrow 0$. Dependence of $\epsilon_{\text {crit }}$ and $\Delta P_{\text {crit }}$ on $\phi$ is not strong (within a factor of 2 between the minimum and the maximum values for a given $\theta$ ).

Critical parameters for contraction exhibit vastly different behaviour. The critical deformation increases monotonically with increase of $\theta$. Dependence on $\phi$ is also mono- 


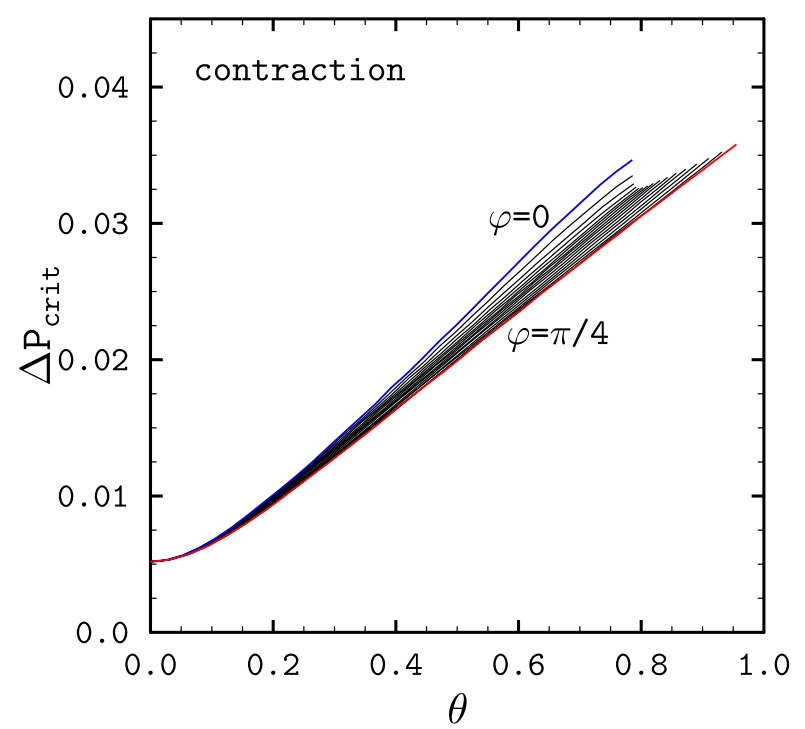

Figure 4. Dimensionless critical pressure anisotropy for contraction.

tonic. For instance, $\epsilon_{\text {crit }} \approx-0.04\left(\xi_{\text {crit }} \approx 0.935\right)$ at $\theta=0$ (and arbitrary $\phi$ ), but at $\theta \sim 0.8$, one has $\epsilon_{\text {crit }} \approx-0.07$ $\left(\xi_{\text {crit }} \approx 0.895\right)$ for $\phi=0$ and $\epsilon_{\text {crit }} \approx-0.05\left(\xi_{\text {crit }} \approx 0.92\right)$ for $\phi=\pi / 4$. It is worth emphasising, that, for contractions, minimum $\left|\epsilon_{\text {crit }}\right|$ obtained here is 2.5 times below ${ }^{2} \epsilon_{\text {crit }}^{\mathrm{MD}} \approx 0.1$. The critical pressure anisotropy increases with increase of $\theta$ (from $\Delta P_{\text {crit }} \approx 0.005$ at $\theta=0$ to $\Delta P_{\text {crit }} \approx 0.035$ at $\theta \sim 1$ ). The dependence on $\phi$ is also not very strong (up to $15 \%$ between the minimum and the maximum values). For all $\theta$ and $\phi$, the crystal breaks at lower pressure anisotropy than the critical $\Delta P^{\mathrm{MD}} \approx 0.039$.

For $\theta=0, \Delta P_{\text {crit }} \sim 0.005$ for both elongation and contraction, but for other stretching directions, the lattice is generally asymmetric for elongation/contraction.

\subsection{Elastic energy}

Stretching the crystal (e.g., by evolving magnetic field) requires work against the elastic forces and increases the crystal electrostatic energy. The latter can be easily computed using the same Ewald method as that employed for the stress tensor calculation ${ }^{3}$. The excess energies at critical deformations are shown in Figs. 5 and 6 for elongation and contraction, respectively, as functions of the same angles. Similar to $\Delta P_{\text {crit }}, \Delta U_{\text {crit }}$ is strongly anisotropic and demonstrates a monotonic behaviour as a function of $\theta$ only for contraction. For elongation, the minimum of $\Delta U_{\text {crit }}$ at $\theta=0$ is less pronounced than the minimum of $\Delta P_{\text {crit }}$. This is due to strongly non-linear elasticity at $\theta \approx 0$ (see Fig. 1 of Paper

2 There is no direct contradiction between these data, because, being presented in eigen-axes $1^{\prime} 2^{\prime} 3^{\prime}$, the shear deformation $\left(u_{12}=\right.$ $\left.u_{21}=\epsilon / 2, \quad u_{33}=0\right)$ corresponds to $u_{1^{\prime} 1^{\prime}}=\epsilon / 2, u_{2^{\prime} 2^{\prime}}=$ $-\epsilon / 2, \quad u_{3^{\prime} 3^{\prime}}=0$. That is, it corresponds to a deformation anisotropic in the plane, perpendicular to the stretch axis. Such deformations are beyond the scope of the present paper.

${ }^{3}$ Non-linear behaviour of the stress-strain curve before the loss of stability (see Fig. 1 in Paper 1) does not allow one to apply the simple linearised theory to calculate the elastic energy precisely.

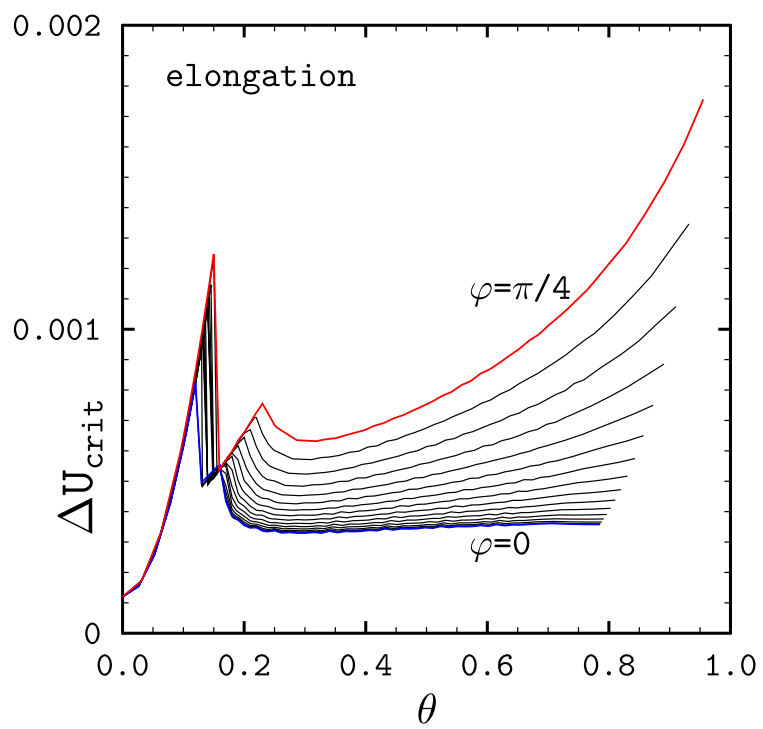

Figure 5. Dimensionless excess electrostatic energy per ion at critical elongation.

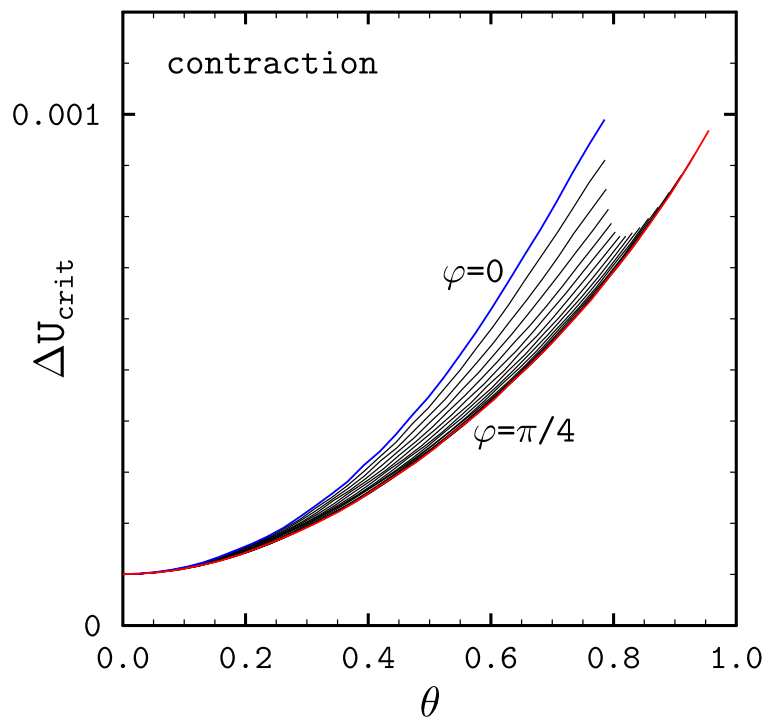

Figure 6. Dimensionless excess electrostatic energy per ion at critical contraction.

1). Let us point out, that for all considered deformations, $\Delta U_{\text {crit }}$ is insufficient to completely melt the crystal, even for a crystal that is already at melting temperature, $T_{\mathrm{m}}$, because the latent heat for a Coulomb crystal is expected to be greater $\left(\sim 0.75 k T_{\mathrm{m}} \sim 0.004 Z^{2} e^{2} / a\right.$ neglecting quantum effects, e.g., Hansen 2004).

\section{APPLICATIONS}

\subsection{Polycrystalline matter}

Let us adopt a popular point of view that matter in the NS crust is polycrystalline (e.g., Strohmayer, Ogata, Iyetomi, Ichimaru \& van Horn 1991; Chamel \& Haensel 2008). Although the validity of this picture is not guaranteed, we defer a discussion of an alternative point of view till Subsection 3.4. Caplan et al. 
(2018) discuss crystallisation of matter in accreting NS and argue that the typical size of crystallites at the crust top should be comparable with the scale height $\sim 10 \mathrm{~m}$. Subsequent compression by pressure of newly accreted material should decrease crystallite (grain) size (if there is no merging between them - see Subsection 3.3), producing a polycrystal with even smaller grains.

If crystallites are randomly oriented and are much smaller than the typical scale of stress and strain variation, the infinitesimal deformations of the polycrystal can be described by the isotropic linear theory of elasticity, in which a material is characterised by just two quantities, the bulk modulus $K$ and the effective shear modulus $\mu_{\mathrm{eff}}$. However, these quantities depend on the correlations between the orientations of the neighbouring crystallites, and, in general, cannot be accurately calculated from elastic properties of a monocrystal (e.g., Landau et al. 1986).

Several ways to determine $\mu_{\text {eff }}$ have been proposed (see, e.g., Kobyakov \& Pethick 2015 for review) with numerical results diverging by as much as a factor of 2.34 assuming bcc crystallites and neglecting electron screening. In NS literature, one typically uses the effective shear modulus $\mu_{\mathrm{eff}}^{\mathrm{V}} \approx 0.1194$ (times $n Z^{2} e^{2} / a$ in physical units) introduced by Ogata \& Ichimaru (1990) on the basis of an angular averaging of the shear-wave velocity. The same quantity is obtained if one assumes a uniform strain in all crystallites and is known as the Voigt average. If, on the contrary, a uniform stress in all crystallites is assumed, one derives the Reuss average $\mu_{\mathrm{eff}}^{\mathrm{R}} \approx 0.051$. Kobyakov \& Pethick (2015) have proposed another value, based on the self-consistent theory of Eshelby $(1961)^{4}$.

\subsection{Breaking properties of polycrystalline matter}

The maximum deformation, which can be elastically supported by the polycrystalline crust, is even more uncertain. Previously, various authors (e.g., Ushomirsky et al. 2000; Johnson-McDaniel \& Owen 2013) applied the von Misses criterion by introducing $\sigma^{2} \equiv \hat{\sigma}_{i j} \hat{\sigma}^{j i} / 2$, where $\hat{\sigma}_{i j}=\sigma_{i j}-$ $\sigma_{l l} \delta_{i j} / 3$ was the traceless stress tensor, and assumed that the critical value of $\sigma$ was $\sigma_{\max }^{\text {trad }} \approx \mu_{\text {eff }}^{\mathrm{V}} \epsilon_{\text {crit }}^{\operatorname{trad}}$. Following Horowitz \& Kadau (2009), who have performed the first direct MD simulations of the breaking strain for the shear deformation, $\epsilon_{\text {crit }}^{\text {trad }}$ was typically set equal to $\epsilon_{\text {crit }}^{\mathrm{MD}} \approx 0.1$ (e.g., Li et al. 2016). Horowitz \& Kadau (2009) have performed simulations not only for monocrystals, but have also made one simulation for a polycrystal and several simulations for monocrystals with defects. They have concluded that the resulting critical strains were only modestly reduced in comparison with $\epsilon_{\text {crit }}^{\mathrm{MD}}$.

Our analysis indicates that, for monocrystals, the breaking parameters are strongly anisotropic and cannot be described in terms of a single number. But what does this

\footnotetext{
${ }^{4}$ For strain $|\epsilon| \lesssim 0.04$ the stress-strain curve for the polycrystalline MD simulation in Fig. 1 of Horowitz \& Kadau (2009) agrees well with the results of Ogata \& Ichimaru (1990), but not with those of Kobyakov \& Pethick (2015). However, this can be a specific property of this particular polycrystal. Note, that for larger $|\epsilon|$, the stress-strain curve becomes strongly non-linear, which can be an evidence of yield.
}

tell us about polycrystalline crust? Just like for infinitesimal deformations, an accurate answer requires modelling the stress/strain distribution inside crystallites and depends on their specific geometry and mutual orientation. However, a detailed solution like that is simultaneously very hard to get and not very useful in the astrophysical context. Instead, let us rely on the same "smoothed-out" models as in Subsection 3.1. As discussed by Kocks (1970), these models are not fully self-consistent, however, the first one (uniform strain, Voigt) results in an upper bound for the strength, whereas the second one (uniform stress, Reuss) gives the lower bound, provided that the yield or breaking is associated with a loss of stability inside one of the crystallites.

In the first approach, the strength must be determined by the minimum (over the stretch directions) of the absolute value of the critical strain, $\left|\epsilon_{\text {crit }}\right| \approx 0.04$ (for elongation it corresponds to $\theta \approx 0.8$ and $\phi \approx 0$, Fig. 1, for contraction it occurs at $\theta \approx 0$ and any $\phi$, Fig. 2). For larger strain, a suitably oriented crystallite will lose stability and the deformation will become inelastic. Accordingly, we expect that the critical strain for elongation/contraction in NS crust cannot exceed 0.04. Respective estimate of the critical pressure anisotropy for the polycrystal is $\Delta P_{\text {crit }}^{\mathrm{V}} \approx$ $3 \mu_{\mathrm{eff}}^{\mathrm{V}} \epsilon_{\mathrm{crit}} \approx 0.014$ corresponding to the von Misses parameter $\sigma_{\text {max }}^{\mathrm{V}} \approx \sqrt{3} \mu_{\text {eff }}^{\mathrm{V}} \epsilon_{\text {crit }} \approx 0.008$ [it is easy to see, e.g., from Eq. (4.6) of Landau et al. 1986 that in the linear regime $\Delta P=\sqrt{3} \sigma=3 \mu \epsilon$ for a stretch of an isotropic body with the shear modulus $\mu$.

The von Misses parameter $\sigma_{\max }^{\mathrm{V}}$ just obtained is 1.5 times lower than the traditional estimate above: $\sigma_{\max }^{\operatorname{trad}} \approx$ 0.0119 . At the same time, it is in a surprisingly ideal agreement with the maximum dimensionless stress $\sigma_{12, \max }^{\mathrm{pMD}} \approx$ 0.008 achieved in the polycrystalline MD simulation of Horowitz \& Kadau (2009) [for the shear deformation of the isotropic body in the linear regime $\Delta P=2 \sigma=2 \mu \epsilon$, while the component $\sigma_{12}$ equals $\sigma$ ].

In terms of the pressure anisotropy, $\Delta P_{\text {crit }}^{\mathrm{V}} \approx 0.014$ is slightly lower than the critical pressure anisotropy inferred from the polycrystalline $\mathrm{MD}, \Delta P_{\mathrm{crit}}^{\mathrm{pMD}} \approx 2 \sigma_{12, \max }^{\mathrm{pMD}} \approx 0.016$; is 1.7 times lower than the traditional estimate, $\Delta P_{\text {crit }}^{\text {trad }} \approx$ $2 \sigma_{\max }^{\text {trad }} \approx 0.0238 ;$ and is 2.8 times lower than $\Delta P^{\mathrm{MD}} \approx 0.039$ for the monocrystal.

In the second approach, if $\Delta P$ exceeds 0.005 , which is the minimum critical pressure anisotropy $\left(\Delta P_{\text {crit }} \approx 0.005\right.$ at $\theta \approx 0$ and any $\phi$ for both elongation and contraction, Figs. 3 and 4 ), the crystallite with a suitable alignment will break. The quantity $\epsilon_{\text {crit }}^{\mathrm{R}} \approx 0.005 /\left(3 \mu_{\mathrm{eff}}^{\mathrm{R}}\right) \approx 0.03$ is the respective estimate of the critical strain.

Notice, that the critical strains predicted by the two approaches are in a good agreement with each other suggesting that $\epsilon_{\text {crit }} \approx 0.03-0.04$ is a rather robust bound for the elastic stretching deformation of the polycrystalline crust.

Let us conclude this part by reminding that effects of electron screening and ion motion will further reduce the critical deformation parameters.

\subsection{Plastic motion}

Finally, let us discuss mechanisms of inelastic deformation in polycrystalline crust. Firstly, the loss of stability and destruction of a small-scale crystallite does not necessarily con- 
stitute a catastrophic event because the energy release will be low (cf. Subsection 3.4). The affected ions may recrystallise and the polycrystal may survive, but become plastically deformed. Secondly, due to anisotropy of the elastic properties, deformation of neighbouring grains with the same strain will lead to different elastic energy (per ion) in different grains (see Figs. 5 and 6 for elastic energy at maximum stable deformation). On top of that, boundary conditions may require a density variation at the grain boundary, due to which the scale of the electrostatic energy as well as the energy of the degenerate electron gas in those grains will also be different. It can make growth of grains with lower total energy at the expense of those with higher energy thermodynamically favourable and lead to plastic motion. Note, that the stress-induced grain growth is known for terrestrial materials and it can be a mechanism of plastic flow (e.g., Rupert, Gianola, Gan \& Hemker 2009).

Other mechanisms of plastic motion (e.g., grain boundary sliding, rotation and merging of grains, see Masuda, Kanazawa, Tobe \& Sato 2018) are also not excluded. Thus, the dominating mechanism of the plastic motion in the NS crust is still highly uncertain and requires additional modelling.

\subsection{Heat source}

Crystallisation of NS crust is an extremely slow and uniform process with plenty of time to anneal out any defects. Additionally, if a large-scale sufficiently strong magnetic field is present, crystallisation may proceed in a way that favours a particular orientation of crystal axes with respect to the magnetic field (Baiko 2011). As discussed in Subsection 3.3, merging of the crystallites may occur in the course of the plastic deformation. These conditions may result in a formation of large-scale perfect or close to perfect crystallites. Under the action of stresses (e.g., due to magnetic field), the crystallites will be stretched non-uniformly. If typical scale of the stretch-factor variation becomes smaller than the crystallites' size, the monocrystal theory developed in Section 2 will directly apply.

The sizes of these crystallites in NS crust will be ultimately determined by stresses produced by the evolving magnetic field (Paper 1). When the breaking limit for a crystallite segment is reached, a possible outcome is a destruction of this segment followed by a formation of a new stress-free configuration. This is a mechanism of crystallite size reduction. On the other hand, such a process would be accompanied by release of the extra electrostatic energy in the form of kinetic energy of ions. Our results allow one to calculate the exact amount of energy released in any particular case. For estimates, let us note that according to Figs. 5 and 6 , about $0.0005 Z^{2} e^{2} / a$ per each nucleus can be typically released. Thus the total energy release per event can be estimated as

$$
Q \approx 1.4 \times 10^{38} \rho_{11}^{4 / 3} Z_{44}^{2} A_{126}^{-4 / 3} S_{10} h_{3} \quad \text { erg },
$$

where $\rho_{11}$ is the mass density in units of $10^{11} \mathrm{~g} \mathrm{~cm}^{-3}, A$ is the ion mass number, $Z_{44}=Z / 44, A_{126}=A / 126, S_{10}$ is the destructed area in units of $1 \mathrm{~km}^{2}$ and $h_{3}$ is the height of the destructed volume in units of $10^{3} \mathrm{~cm}$.

In order to produce such a critical stretch at $\rho=10^{11} \mathrm{~g}$ $\mathrm{cm}^{-3}, Z=44$, and $A=126$, one has to have $B \delta B \sim 10^{28} \mathrm{G}^{2}$
(Paper 1). In other words, a magnetic field of, say, $2 \times 10^{14}$ G has to vary by $25 \%$ over the distance of $\sqrt{S} \sim 1 \mathrm{~km}$. This seems realistic for magnetars.

\section{CONCLUSIONS}

In an attempt to deepen understanding of the strength of the NS crust, we have performed a detailed study of uniaxial stretches of crystallites comprising it. The crystallites are modelled as arbitrarily oriented perfect bcc Coulomb crystals. In Section 2 we have demonstrated that the critical strain, above which the crystal lattice lost its stability, was highly anisotropic varying from 0.04 to 0.3 as a function of mutual orientation of the stretch direction and the crystallographic axes. The same holds true for the critical pressure anisotropy (which varies from 0.005 to $0.04 n Z^{2} e^{2} / a$ ) and the excess energy per ion at the critical strain (which may vary from 0.0001 to $0.0018 Z^{2} e^{2} / a$; note that this is insufficient to melt the whole crystallite even if it is already at melting temperature). The high degree of breaking anisotropy implies that the von Misses and Tresca failure criteria are invalid for bcc Coulomb monocrystals.

Our numerical results are presented in Figs. 1-6 in dimensionless form. These data can be converted to physical units with the aid of the scaling relations (2) and (3). All results of Section 2 are based on standard lattice dynamics and are rather robust. However, they are obtained for a pure Coulomb static lattice. We expect that ion motion and electron screening effects would reduce critical deformation parameters by some 10-20\%. The approach of Paper 1 applied here can be used to study the whole five-dimensional yield surface in the six-dimensional space of strains, while in this paper we constrain ourselves to uniaxial deformations only, which span a three-dimensional subspace ${ }^{5}$.

In Section 3, our results for monocrystals are used to estimate the strength of the polycrystalline NS crust. We argue that polycrystalline crust breaking is determined by either the minimum critical strain or the minimum critical pressure anisotropy achievable in monocrystals. This implies the breaking strain for the uniaxial stretch to be $\epsilon_{\text {crit }} \approx 0.03-0.04$, which is a factor of $2.5-3$ lower than the typically assumed value of $\epsilon_{\text {crit }}^{\text {trad }} \approx 0.1$ motivated by MD simulations of a shear deformation. Unlike the breaking strain, the breaking stress is found to be model dependent. The pressure anisotropy at breaking is predicted to lie in the range between 0.005 and $0.014 n Z^{2} e^{2} / a$. This range excludes all the estimates reported previously for the shear deformation: $\Delta P_{\text {crit }}^{\mathrm{pMD}} \approx 0.016$ from the polycrystalline $\mathrm{MD}$, $\Delta P_{\text {crit }}^{\text {trad }} \approx 0.0238$ used traditionally, and $\Delta P_{\text {crit }}^{\mathrm{MD}} \approx 0.039$ from the monocrystalline MD. Alternatively, referring to the von Misses criterion, the critical parameter $\sigma_{\max }$ for stretches is predicted to lie in the range from 0.003 to 0.008 . The upper bound here coincides with $\sigma_{\max }^{\mathrm{pMD}}$ for the shear

5 As discussed in Section 2, a uniform compression should not lead to breaking. Hence, two additional deformation parameters require further research. These can be, for instance, the orientation of the second principal strain axis and the strain along this axis. 
deformation in the polycrystalline MD. The other two estimates for the shear deformation, $\sigma_{\max }^{\text {trad }} \approx 0.0119$ (traditional) and $\sigma_{\max }^{\mathrm{MD}} \approx 0.0195$ (from the monocrystalline MD), fall outside our range.

We also discuss a formation of large crystallites in the NS crust and raise a possibility of plastic flow driven by the stress-induced grain growth. Finally, we calculate heat release from crust breaking events in the context of magnetic field evolution and magnetar flaring activity. We would like to emphasise that Section 3 is of somewhat speculative character. However, its predictions can, in principle, be checked with carefully designed MD simulations. We plan to do this subsequently.

\section{ACKNOWLEDGMENTS}

We are grateful to Russian Science Foundation grant 14-1200316 for support.

\section{REFERENCES}

Baiko D. A., 2011, MNRAS, 416, 22

Baiko D. A., 2014, in Journal of Physics Conference Series. p. 012010, doi:10.1088/1742-6596/496/1/012010

Baiko D. A., 2015, MNRAS, 451, 3055

Baiko D. A., Kozhberov A. A., 2017, MNRAS, 470, 517

Caplan M. E., Horowitz C. J., 2017, Rev. Mod. Phys., 89, 041002

Caplan M. E., Cumming A., Berry D. K., Horowitz C. J., Mckinven R., 2018, preprint, (arXiv:1804.06942)

Chamel N., Haensel P., 2008, Liv. Rev. Relativ., 11, 10

Chugunov A. I., Horowitz C. J., 2010, MNRAS, 407, L54

Chugunov A. I., Horowitz C. J., 2012, Contributions to Plasma Physics, 52, 122

Eshelby J. D., 1961, Prog. Solid Mech., 2, 87

Gabler M., Cerdá-Durán P., Stergioulas N., Font J. A., Müller E., 2018, MNRAS, 476, 4199

Haensel P., Potekhin A., Yakovlev D., 2007, Neutron Stars 1: Equation of State and Structure. Astrophysics and Space Science Library, Springer

Hansen B., 2004, Phys. Rep., 399, 1

Haskell B., Patruno A., 2017, Physical Review Letters, 119,161103

Hoffman K., Heyl J., 2012, MNRAS, 426, 2404

Horowitz C. J., 2010, Phys. Rev. D, 81, 103001

Horowitz C. J., Kadau K., 2009, Physical Review Letters, 102,191102

Johnson-McDaniel N. K., 2013, Phys. Rev. D, 88, 044016

Johnson-McDaniel N. K., Owen B. J., 2013, Phys. Rev. D, 88,044004

Kobyakov D., Pethick C. J., 2015, MNRAS, 449, L110

Kocks U. F., 1970, Metallurgical Transactions, 1, 1121

Landau L., Lifshitz E., Kosevich A., Sykes J., Pitaevskiǔ L., Reid W., 1986, Theory of Elasticity. Course of theoretical physics, Butterworth-Heinemann, https://books.google.ru/books?id=tpY-VkwCkAIC

Lander S. K., 2016, ApJ, 824, L21

Li X., Levin Y., Beloborodov A. M., 2016, ApJ, 833, 189

Masuda H., Kanazawa T., Tobe H., Sato E., 2018, Scripta Materialia, 149, 84

Ogata S., Ichimaru S., 1990, Phys. Rev. A, 42, 4867

Regel' V. R., Slutsker A. I., Tomashevskii É. E., 1972, Soviet Physics Uspekhi, 15, 45

Rupert T. J., Gianola D. S., Gan Y., Hemker K. J., 2009, Science, 326,1686
Strohmayer T., Ogata S., Iyetomi H., Ichimaru S., van Horn H. M., 1991, ApJ, 375, 679

Tsang D., Read J. S., Hinderer T., Piro A. L., Bondarescu R., 2012, Physical Review Letters, 108, 011102

Ushomirsky G., Cutler C., Bildsten L., 2000 , Monthly Notices of the Royal Astronomical Society, 319, 902 